\title{
Muntjaks (Muntiacus reevesi) in Schleswig-Holstein - Beginn einer Invasion, erfolgreiche Bekämpfung oder beides ein bisschen?
}

\author{
Muntjacs in Northern Germany - start of an invasion, successful \\ management or both a bit?
}

\author{
Björn Schulz, Peter Borkenhagen \\ Faunistisch-Ökologische Arbeitsgemeinschaft (FÖAG) e.V., c/o Institut für Natur- und Ressourcenschutz der Christian- \\ Albrechts-Universität zu Kiel, Olshausenstraße 75, 24118 Kiel, bjoern@foeag.de, borkenhagen.sag@t-online.de
}

\section{Schlüsselwörter:}

Neobiota, Aussetzung, SchleswigHolstein, Jagd, Ausrottung
Keywords:

invasive animal, release, Schleswig-Holstein, hunt, eradication

\begin{abstract}
Zusammenfassung
In Schleswig-Holstein treten seit 2015 immer wieder Individuen der als invasiv eingestuften Chinesischen Muntjaks auf. Das Geschehen um die mutmaßlich illegal freigelassene Population bei Kosel im Kreis Rendsburg-Eckernförde konnte von den Autoren intensiver begleitet werden. Sie beschreiben die möglichen Hintergründe einer Freilassung, den Verbleib der Tiere, dabei bisher eingesetzte Fangmethoden und vorstellbare weitere Methoden sowie die potenziellen ökologischen Auswirkungen von Muntjaks und die rechtlichen Hintergründe.
\end{abstract}

\begin{abstract}
In Schleswig-Holstein repeatedly invasive Chinese muntjacs have been documented since 2015. The authors describe especially the fate of a small population near Kosel (district of Rendsburg-Eckernförde), which was presumably illegally released. They further describe the possible background of the release, the fate of single animals, the use of trapping methods as well as the potential ecological effects of muntjacs and finally the legal background.
\end{abstract}

\section{Einleitung}

Muntjaks gehören zu einer Gattung von Hirschartigen, die sich durch ihre geringe Körpergröße (bis $33 \mathrm{~kg}$, nur Riesenmuntjaks Muntjacus vuquangensis auch bis $50 \mathrm{~kg}$ ), durch ein höchstens einfach verzweigtes und kleines Geweih mit hohen Rosenstöcken sowie den aus dem Maul ragenden Eckzähnen auszeichnen. Ihr natürliches Verbreitungsgebiet ist auf das südöstliche Asien beschränkt. Zu dieser Gattung gehören nach Burgin et al. (2020) 13, nach Groves \& Grubb (2011) 16 Arten, von denen einige erst in jüngerer Zeit entdeckt und beschrieben wurden. Der Chinesische Muntjak oder Reeves‘ Muntjak (Muntjacus reevesi Ogilby 1839) ist die Muntjak-Art mit dem größ- ten Verbreitungsgebiet. Neben dem natürlichen Areal im südöstlichen China (Timmins \& Chan 2016) gibt es heute angesiedelte Populationen dieser Art im gesamten Vereinigten Königreich (nach Dick et al. 2009 zusätzlich auf Irland), dessen Gründerpopulation in Südmittelengland im Jahr 1901 zusammen mit den später wieder verschwundenen Indischen Muntjaks (Muntjacus muntjacus) freigelassen wurde (Chapman 1996). Auch in Frankreich wurden von 2013 bis heute mehrere Funde gemeldet, so dass dort mit etablierten Beständen gerechnet werden muss (Blottiere 2018). Auf dem europäischen Festland sind den Autoren keine weiteren Populationen bekannt, die über Jahre Bestand hatten oder in denen Freilandreproduktion nachgewiesen werden konnte. In der umfangreichen Darstellung 


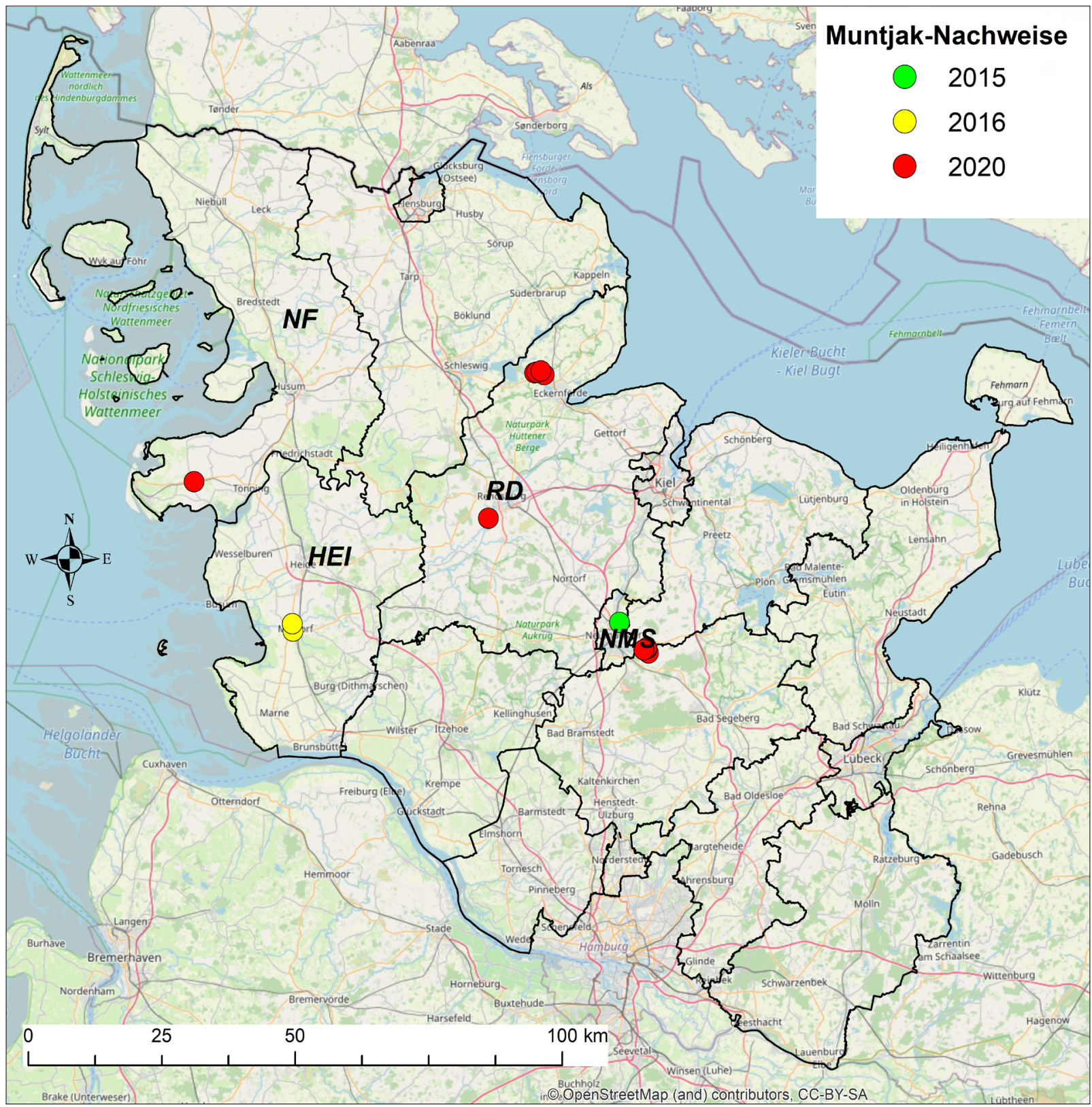

Abb. 1: Muntjak-Nachweise der jüngeren Vergangenheit in Schleswig-Holstein (Punktansammlung im nördlichen Kreis RD zwischen Eckernförder und Schleswig = Koseler Bestand, Punktansammlung östlich von NMS = Kummerfelder Bestand).

des CABI (2021) umfasst die Verbreitungskarte neben den Britischen Inseln Frankreich, Niederlande, Belgien und Korsika.

Muntjaks müssen nach der EU-Verordnung 1143/2014 in den Mitgliedsstaaten der EU aufgrund der von ihnen ausgehenden Gefährdung der Biodiversität sofort aus der Natur entnommen werden - nur im Falle einer bereits weiten Verbreitung unterlägen sie dem einfachen Management. Im Vergleich $\mathrm{zu}$ anderen invasiven $\mathrm{Ar}$ ten, die sich bereits an vielen Stellen Europas etabliert haben, scheint die Ausrottung der wenigen bekannten
Muntjak-Populationen noch möglich zu sein, sofern keine wesentlichen Fehler gemacht werden oder Versäumnisse eintreten.

Aktuelle Daten aus Schleswig-Holstein zeigen, dass es - anders als die derzeit gültige Rote Liste der Säugetiere (Meinig et al. 2020) darstellt - bereits seit mehreren Jahren bestehende und vermutlich reproduzierende Freilandpopulationen in Deutschland gibt. Es muss von einer akuten Invasionsgefährdung ausgegangen werden, was schnelles und entschiedenes Handeln erforderlich macht. Anhand von zwei Lokalitäten mit 
Tab. 1: Muntjak-Nachweise in Schleswig-Holstein.

\begin{tabular}{|c|c|c|c|c|c|}
\hline Jahr & Zeitraum & Ort & Kreis & n & Beschreibung \\
\hline 2015 & 5.9 . & Neumünster & NMS & 1 & Sichtmeldung mit Foto \\
\hline \multirow[t]{2}{*}{2016} & $\begin{array}{l}\text { Feb.-März } \\
\text { Herbst }\end{array}$ & Meldorf & $\mathrm{HEI}$ & 1 & $\begin{array}{l}\text { div. Fotonachweise } \\
\text { Fund von totem Ind. nach Verkehrsunfall }\end{array}$ \\
\hline & 14.7. & Neumünster & NMS & 1 & Sichtmeldung mit Foto \\
\hline \multirow[t]{6}{*}{2020} & $\begin{array}{l}28.1 . \\
\text { seit } 23.3 .\end{array}$ & $\begin{array}{l}\text { Arnis } \\
\text { Kosel }\end{array}$ & $\mathrm{SL}$ & $\begin{array}{l}1 \\
\geq 10\end{array}$ & $\begin{array}{l}\text { Todfund } \\
\text { Entnahme \& Fang andauernd; bis Nov. } 8 \text { Ind. } \\
\text { entweder tot aufgefunden oder lebend gefangen }\end{array}$ \\
\hline & 15.4. & $\begin{array}{l}\text { Vogelsang- } \\
\text { Grünholz }\end{array}$ & $\mathrm{RD}$ & 1 & Sichtmeldung mit Foto \\
\hline & seit 12.7 & Kummerfeld & SE & $\geq 4$ & $\begin{array}{l}\text { Entnahme andauernd, } 2 \sigma^{7} \sigma^{7}, 2 \% \text { @ nach Entnahme- } \\
\text { genehmigung von Jägern erlegt: „Vorkommen seit } \\
\text { Jahren bekannt" }\end{array}$ \\
\hline & 31.7. & Tating & NF & 1 & $10^{\pi}$ nach Entnahmegenehmigung von Jäger erlegt \\
\hline & 28.9. & Schülp & $\mathrm{RD}$ & 1 & $\begin{array}{l}10^{\top} \text { ertrunken aus dem Nord-Ostsee-Kanal (NOK) } \\
\text { geborgen, am gleichen Ort Sichtung am 13.9. }\end{array}$ \\
\hline & 5.10. & Rickert & $\mathrm{RD}$ & 1 & Sichtung \\
\hline
\end{tabular}

individuenreicheren Muntjak-Beständen wird das im Sommer 2020 begonnene Beseitigungsvorhaben von Muntjaks in Schleswig-Holstein näher beleuchtet und diverse Hintergründe werden diskutiert.

\section{Methoden und Ergebnisse}

Die Autoren haben anhand einer Internet- und Literaturrecherche, der Daten des Landesartenkatasters (LLUR 2020) und ergänzenden Befragungen vor Ort sowie selbst erhobenen Daten die jüngeren Funde von Muntjaks in Schleswig-Holstein zusammengetragen. Bei den Tieren auf den vorhandenen Bildern handelt es sich mit größter Wahrscheinlichkeit um den Chinesischen Muntjak, Zwerg-Muntjak bzw. Reeves Muntjak (Muntjacus reevesi Ogilby 1839), es haben allerdings keine morphologischen oder genetischen Untersuchungen zur Art-Überprüfung stattgefunden. Im Jahr 2015 wurden freilebende Muntjaks für Schleswig-Holstein gemeldet, die erste Beobachtung fand am 5.9.2015 in einem Privatgarten in den Außenbezirken von Neumünster (NMS) statt und am 26.9.2015 wur- de darüber in der Lokalpresse berichtet (Ziehm 2015). Aus Meldorf (HEI) wurde berichtet, dass ein Muntjak erst monatelang durch Gärten streifte (Boyens Medien 2016, 2017) und dann im Herbst 2016 durch einen Verkehrsunfall ums Leben kam (Behnke, mdl. Mitteilung). Nach diesen Einzelnachweisen in 2015 und 2016 häufen sich in 2020 die Meldungen von Muntjaks (Tab. 1), u.a. auch durch in den Medien verbreitete Aufrufe des LLUR zur Meldung von Muntjak-Sichtungen. Die überprüfbaren Hinweise (durch Fotobeleg, Kadaver oder lebendes Tier) sind in der Abbildung 1 dargestellt, auf die Darstellung von eingegangenen, aber nicht überprüfbaren Hinweisen (analog SCALP-Kriterium C3, vgl. Molinari-Jobin 2003) wurde verzichtet.

\section{Koseler Muntjak-Population}

Am 25.3.2020 wurde den Autoren aus der Region Kosel von einer Privatperson gemeldet, dass sie seit 23.3.2020 mindestens drei Muntjaks in einem privaten Wald beobachten könne, in dem erst kürzlich ein „Gehege“ mit einem aufwändig instand gesetzten Wildschutzzaun und Fütterungseinrichtungen errichtet worden sei und man befürchtete, dass die die Tiere in 
Kürze freigelassen werden sollen. Diese Meldung haben die Autoren umgehend an die zuständige Obere Naturschutzbehörde (LLUR) weitergegeben.

Seit dieser ersten Meldung im März 2020 sind viele weitere (auch eigene) Bildbelege, Sichtmeldungen ohne Beleg, aber auch in mobilen Schaf- oder Geflügelnetzen tot aufgefundene oder mit solchen Netzen lebend gefangene und dann in sichere Gehegehaltung überführte Tiere dokumentiert worden. Stand Mitte Oktober 2020 befanden sich acht Tiere in Gefangenschaft und vier weitere Tiere sind tot aufgefunden worden, mehrere Tiere dürften noch freilebend sein.

Anzunehmen ist, dass die Tiere bewusst ausgesetzt worden sind. Die Autoren haben in einem Internet-Tiermarkt ein veröffentlichtes Kaufgesuch vom 21.2.2020 aus dem nahe bei Kosel gelegenen Kappeln gefunden „suche Muntjaks m/w zu kaufen zur tiergerechten Haltung in weitläufigem Gehege. Biete guten Preis. Gern auch Jungtiere“. Es ist nicht unwahrscheinlich, dass es sich bei den freigelassenen Tieren um die per Annonce gesuchten Tiere handelt. Interessant wird sein, ob eine verantwortliche Person ermittelt werden kann und wie dem Satz 33 der EU-Verordnung 1143/205 gefolgt wird, der Sanktionen fordert, die „dem Grundsatz der Kostenerstattung und dem Verursacherprinzip Rechnung tragen.“

\section{Kummerfelder Muntjak-Population}

Den Kummerfelder Jägern sind nach eigenen Angaben freilebende und möglicherweise aus einer ortsbekannten Haltung entflohene und sich auch reproduzierende Muntjaks seit mehreren Jahren bekannt. Sie würden sich bisher sehr ortstreu in einem Umkreis von $1 \mathrm{~km}$ aufhalten, wenngleich in einer Entfernung von $3 \mathrm{~km}$ ein Verkehrsunfall mit Muntjak gemeldet worden sein soll (Bilder davon konnten nicht ermittelt werden), was für einen größeren Aktionsraum oder eine größere Population spräche. Die im Sommer 2020 durch das LLUR ausgegebenen Entnahmegenehmigungen haben innerhalb von kurzer Zeit zum Abschuss von vier Muntjaks geführt, Bilder der erlegten Tiere sind nach jedem Abschuss dem LLUR übermittelt worden. Stand Mitte Oktober 2020 gehen die Jäger von weiteren freilebenden Muntjaks aus.

\section{Diskussion}

\section{Mögliche Herkunft}

Tierparks und Zoos sind als (ehemalige) Halter von Muntjaks bekannt. So hatte z.B. der Tierpark Gettorf 2015 (Kreis Rendsburg) ein Muntjakpärchen von Hagenbecks Tierpark erhalten (Concentus-redivivus. de 2020) und hält nach eigenen Informationen (Tierparkgettorf.de 2020) immer noch Muntjaks, allerdings handelt es sich dabei um Indische Muntjaks (Muntiacus muntjak). Im Kreis Segeberg (SE) ist der Oberen Naturschutzbehörde eine Privathaltung bekannt gewesen, in deren Umfeld es spätere Freilandfunde gab (Drews, mdl. Mitteilung). Schließlich werden Muntjaks trotz eines unionsweiten Handels- und Haltungsverbots auch in Schleswig-Holstein immer wieder zum Kauf angeboten oder von Käufern gesucht:

- Kaufgesuch von Muntjaks vom 21.2.2020 (Quoka. de 2020) aus dem Raum Kappeln (Schleswig),

- Verkaufsangebot von Muntjaks aus dem Raum Neustadt in Holstein (Annoncen.org 2020).

Die Herkunft der Koseler Chinesischen Muntjaks wurde bisher nicht aufgeklärt. Die Herkunft aus Tierparks und Zoo scheint aus o.g. Gründen ausgeschlossen, eine oder mehrere private und ungenehmigte Haltungen und die (absichtliche?) Ansiedlung ist wahrscheinlicher. Aufgrund der räumlichen und zeitlichen Nähe erscheint den Autoren ein Zusammenhang mit dem Kaufgesuch vom 21.2.2020 aus Kappeln naheliegend.

\section{Jagdliches Interesse als möglicher Ansiedlungsgrund}

Muntjaks gelten als ortstreu und zeigen dabei Reviergrößen von wenigen hundert Metern Durchmesser (Chapman et al. 1993), womit sie für eine Ansiedlung in nur kleinen Jagdrevieren interessant sein könnten. Gleichzeitig gelten sie aufgrund ihrer normalerweise versteckten Lebensweise als jagdlich herausfordernd und das Wildbret der Tiere gilt als wertvoll, wenngleich unter Umständen Trichinen-belastet. Sie unterliegen zwar nicht dem Jagdrecht und dürften deshalb von Jägern auch nicht bejagt werden, doch erfolgt im Revier keine Kontrolle und eine Entnahme ist ausdrücklich erwünscht und wird deshalb auf Antrag durch die zuständigen Behörden schnell gestattet. Das Vorkommen von Muntjaks in einem Revier könnte für den Jagdrevierinhaber auch deshalb interessant sein, weil sie nicht wildschadensersatzpflichtig sind durch Muntjaks entstandene Schäden muss ein Jagdausübungsberechtigter gegenüber dem Geschädigten 

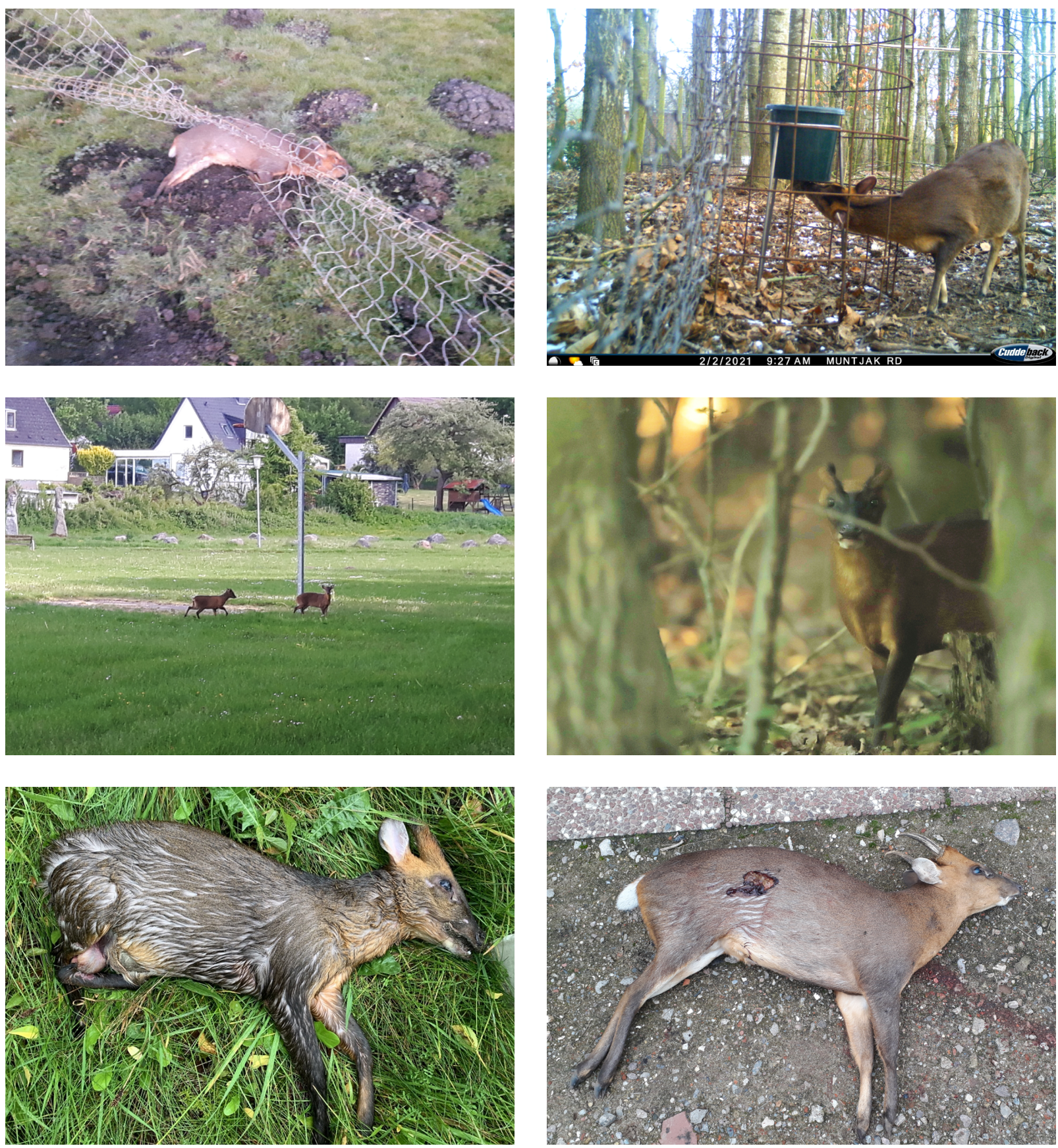

Abb. 2: Links oben: ein bei Kosel (RD) im Schafsnetz verendetes Muntjak (26.3.2020); rechts oben: mit Baustahlmatten geschützte Futtereimer sind für Muntjaks leicht nutzbar (1.2.2021, in legaler Gehegehaltung); links mittig: ortsnahe Aufenthaltsorte von entlaufenen Muntjaks scheinen sich besonders für den Fang mit Hilfe von Zaunanlagen zu eignen (Kosel, 24.5.2020); rechts mittig: eines der noch freilaufenden Tiere (mit entfernten Ohren? Kosel, 25.6.2020); links unten: das bei Schülp (RD) aus dem NOK tot geborgene (säugende) Tier (28.9.2020); rechts unten: ein bei Kummerfeld (SE) erlegtes Muntjak (30.9.2020).

nicht ersetzen (siehe entsprechende Forumsdiskussion auf WildundHund.de 2018). Solche Regelungen können vom Gesetzgeber schnell nachgeholt werden, wie die Aufnahme der Neozoen Marderhund, Waschbär, Mink und Nutria in das Jagdgesetz als jagdbare
Wildarten (LJagdG SH 1999) zeigt. Das jagdliche Interesse an Muntjaks kann z.B. durch Jagdzeitschriften geweckt werden, denn Anbieter von Jagdreisen bewerben die kostenpflichtige Jagd auf Muntjaks und das Wasserreh (Hydropotes inermis) auf den briti- 
schen Inseln z.B. mit dem Verweis auf die ungewöhnlichen Trophäen („Eckzähne, die seine Trophäe aussehen lassen, als käme sie aus einer ganz anderen - und viel dunkleren - Welt“, Diana-Jagdreisen.de 2020).

Möglich scheint, dass im nordöstlichen Schleswig-Holstein die Idee der Bereicherung von Jagdrevieren durch eingeführte Tierarten besonders verbreitet und damit die Hemmschwelle zur Ansiedlung weiterer Arten besonders niedrig ist. Im dortigen Umfeld sind größere und teils langjährig etablierte Vorkommen von eingeführten jagdbaren Arten wie Damhirsch (Dama dama), Sikahirsch (Cervus nippon) und Mufflons (Ovis gmelini) (Borkenhagen 2011, Wildtierkataster SH 2020) in teilweise bemerkenswert hohen Dichten (Greiser et al. 2020) vorhanden. Zudem kommen weitere jagdbare Neozoen wie Waschbär, Marderhund, Mink, Nutria oder Jagdfasan vor, während einheimische Arten wie Rothirsch (Cervus elaphus) nach ministerieller Erlasslage nicht vorkommen dürfen. Derzeit „unterdrücken“ die politisch-(jagd-)rechtlichen Rahmenbedingungen die einheimischen Arten, während das vorhandene Gefüge von Neozoen und Neuansiedlungen faktisch geduldet wird, wodurch möglicherweise ein „falscher“ Gewöhnungseffekt eingetreten ist („shifting baseline“, Clavero 2014). Aus ökologischer und wildtierbiologischer Sicht sind hier Anpassungen und effektive Handlungsstrategien sowie Aufklärungskampagnen pro einheimische Arten und contra invasive Arten am besten in Kooperation mit der aufgeklärten Jägerschaft dringend überfällig.

\section{Fangmethoden}

Mehrere der Koseler Tiere konnten in menschliche Obhut gelangen, nachdem sie sich in Geflügel- oder Schafsnetzen (,Euro-Netze`) verfangen hatten. Anfänglich geschah dies zufällig und ausschließlich an bestehenden Einzäunungen, ab Ende Juni hat einer der Autoren Schafnetze an die lokalen Helfer geliefert um deren Fangerfolg zu erhöhen. Durch Aufstellen an besonders geeigneten Stellen in Siedlungsnähe konnten die lokalen Helfer bis Oktober 2020 insgesamt fünf weitere Tiere unversehrt einfangen. Auch Chapman et al. (1987) betonen die Bedeutung von Netzfängen in britischen Vorkommensgebieten. Dort wurden die einige $100 \mathrm{~m}$ langen Netze jedoch zunächst flach ausgelegt. Mit Hilfe von geschultem Personal wurden Muntjaks in die Netze geleitet, durch Hochheben der Netze gefangen und zur Vermeidung von Verletzungen sofort befreit bzw. in auf Muntjak-Bedürfnisse abgestimmte Aufbewahrungsboxen überführt. Casaer et al. (2015) diskutieren neben den Lebendfangmethoden (Netzfang, Fallenfang) vor allem verschiedene geeignete Abschussmethoden. Baiwy et al. (2013) verweisen darauf, dass Vorkommen von Muntjaks aufgrund ihrer geringen Größe und der versteckten Lebensweise meist erst dann wahrgenommen werden, wenn sie eine größere Population aufgebaut haben. Fangmethoden können ihrer Meinung nach effektiv sein, sollten aber immer an die lokalen Umstände angepasst werden, auch um das Tierwohl nicht zu gefährden. Die umfassendste Übersicht über Erfassungs- und Entnahmemethoden speziell von Muntjaks geben Dick et al. (2009). Auch in SchleswigHolstein sollte sich das Invasionsartenmanagement der effektivsten Methoden bedienen und entsprechende personelle Kompetenzen aufbauen.

\section{Mögliche Probleme durch Muntjaks}

Muntjaks sind frühreif (mit 6 - 12 Monaten), haben ein hohes mögliches Lebensalter (bis 17 Jahre) und sind asaisonal brunftig, d.h. Nachwuchs ist zu allen Jahreszeiten möglich (Chapman et al. 1984, Wagener et al. 2019). Einmal etablierte Bestände können also im Vergleich zu anderen Hirschartigen schnell wachsen. Da bereits junge Weibchen Nachwuchs führen können und vom Muttertier abhängiger Nachwuchs zu jeder Jahreszeit erwartbar ist (der aber nur selten sichtbar sein dürfte), sind an die Entnahmemethoden hohe tierschutzfachliche Anforderungen zu stellen, um das Tierwohl des Jungtieres nicht zu gefährden.

Muntjaks ernähren sich überwiegend phytophag, haben aufgrund ihrer geringen Körpergröße einen besonders hohen Nährstoffbedarf und gehören damit dem „browser-“ oder auch Konzentratselektierer-Typ an. Cooke et al. (2001) haben Auswirkungen von Muntjak-Fraß besonders an als Niederwald genutzten Baumbeständen (Hasel, Feldahorn und Esche) und begleitenden Kräutern beobachtet, während Gräser kaum genutzt wurden. Am ehesten dürfte der Einfluss von Muntjaks also mit dem von Rehen zu vergleichen sein und damit forstwirtschaftlich als potenziell problematisch beurteilt werden. Der Effekt von Muntjaks auf die Vegetation kann zudem einen Effekt auf andere phytophage Tiere haben. So weisen Pollard \& Cooke (1993) daraufhin, dass Muntjaks die wichtigste Futterpflanze des Kleinen Eisvogels (Limenitis camilla L. 1764) ausgerechnet dort bevorzugt befressen, wo die Schmetterlinge ihre Eier ablegen, wodurch es zu einem dortigen Bestandsrückgang des auch in Schleswig-Holstein sehr seltenen Tagfalters (in SH RL 1, Kolligs 2003) gekommen ist. 
Muntjaks sind keine reinen Pflanzenfresser, sondern ernähren sich ergänzend von lebenden und toten Tieren. Da sie kleine Wirbeltiere erbeuten können oder Vogeleier zu sich nehmen, ist innerhalb der Muntjak-Reviere von einer bislang nicht näher quantifizierbaren zusätzlichen Prädation der Bodenfauna und von Bodenbrütergelegen auszugehen.

Wie alle Säugetiere dürften auch Muntjaks Reservoire einer großen Zahl von Viren- und Bakterien-Arten sein und eine Fülle von Endo- und Ektoparasiten beherbergen und verbreiten. Wenngleich der Wissensstand hierüber sehr niedrig ist, sind sie dennoch als Überträger verschiedener Krankheiten bekannt, die auf einheimische Arten übertragen werden können (Wagener et al. 2019).

Im Vergleich zum ökologisch ähnlich eingenischten Reh könnten sich Muntjaks als konkurrenzstärker erweisen. So wird aus dem Vereinigten Königreich berichtet, dass Muntjaks in größeren Dichten vorkommen als Rehe (Dolman \& Wäber 2008). Nicht zuletzt wegen dieser großen Dichte gehören Muntjaks in Teilen Englands zu den häufigsten Verursachern bzw. Opfern von wildbedingten Verkehrsunfällen (Langbein 2007).

\section{Zukunft der Lokalpopulationen}

Die Koseler Muntjak-Population wurde bis Oktober 2020 mehr als ein halbes Jahr beobachtet. In diesem Zeitraum konnten zwar immer wieder Tiere eingefangen und sicher verwahrt bzw. tote Tiere eingesammelt werden, gleichzeitig wurden aber immer wieder auch Sichtungen von weiteren im Freiland lebenden Tieren gemeldet. Zudem hat eines der gefangenen Weibchen kurz nach dem Fang ein Kitz gesetzt, so dass Reproduktion im Freiland wahrscheinlich ist. Versuche $\mathrm{zu}$ Ermittlung des noch freilebenden Bestandes haben nicht stattgefunden, sind nach Hemami et al. (2007) nicht einfach und die Nutzung von z.B. Wärmebildoptik wird empfohlen.

Freeman et al. (2015) haben innerhalb des bekannten Verbreitungsgebietes auf den britischen Inseln Proben von 176 Muntjak-Individuen genetisch untersucht und kommen zum Schluss, dass die gesamte Invasion der Art letztlich auf ein einzelnes Ansiedlungsereignis von nur wenigen Tieren (vier bis fünf Weibchen) und vermutlich anschließend mehrfachen aktiven Verfrachtungen zurückgeführt werden kann. Die Population in Kosel wurde von den Beteiligten zwischenzeitlich auf mehr als 20 Tiere geschätzt, Stand Oktober 2020 befanden sich acht Tiere in Gefangenschaft und vier Tiere waren bis dahin verstorben und einige Tiere liefen noch frei herum. Nach den Ergebnissen von Freeman et al. (2015) kann die verbliebene Populationsgröße ausreichend sein, um sich zu etablieren und trotz der mutmaßlich geringen genetischen Vielfalt die Gründerpopulation für eine umfassende Besiedlung nicht nur Norddeutschlands darstellen. Die nahe Zukunft wird zeigen, ob die seitens der Landesbehörden für die Beseitigung der Population verwendeten Mittel und Werkzeuge ausreichend sind.

Wie hoch die Chancen auf ein natürliches Verschwinden der Population sind, kann nicht quantifiziert werden. Es sind wenige natürliche Faktoren bekannt, die einen Bestand reduzieren könnten. So beobachteten Cooke et al. (1996) die Reduktion einer britischen Lokalpopulation um 70 \% während des besonders harten Winters 1962/63 durch Erfrieren, Verhungern und Prädation durch Hunde.

\section{Rechtliche Situation}

Maßgeblich für die rechtliche Handhabung von freilebenden Muntjaks ist die „Verordnung (EU) Nr. 1143/2014 des europäischen Parlaments und des Rates vom 22. Oktober 2014 über die Prävention und das Management der Einbringung und Ausbreitung invasiver gebietsfremder Arten“. Sie wurde in deutsches Recht übertragen durch das „Gesetz zur Durchführung der Verordnung (EU) Nr. 1143/2014 über die Prävention und das Management der Einbringung und Ausbreitung invasiver gebietsfremder Arten vom 8. September 2017“. In der Folge sind das BNatSchG (um §40a ff.) und das BJagdG (um §28a) ergänzt worden, in denen die Entnahme von invasiven Arten geregelt wird. Danach ist der Jagdausübungsberechtigte (JAB) gemäß Jagdrecht am Management der invasiven Arten zu beteiligen (sofern dieses möglich und wirksam ist), er ist aber nicht zur Durchführung verpflichtet. Die Jagdbehörde kann jagdliche Maßnahmen auch selbst übernehmen oder einen Dritten beauftragen - bisher allerdings nur im Einvernehmen mit dem JAB, was von den Autoren als sehr kritisch angesehen wird, da dieses eine schnelle und effektive Entnahme behindern könnte.

(Ergänzung: Mit Beschluss des OVG Schleswig vom 15.9.2021 ist bei Vorkommen von Muntjak sofortiges Handeln geboten und damit das Einvernehmen mit dem JAB entbehrlich, (AZ 5 MB 22/21).)

\section{Effektivität der verschiedenen ,Beseitigungsaktionen' in Schleswig-Holstein}

Anhand von mehreren Beispielen aus Schleswig-Holstein kann gezeigt werden, dass bei der effektiven 
Tab. 2: Ergebnisse der Bemühungen zur Beseitigung von Muntjak-Vorkommen in Schleswig-Holstein (Stand: Ende 2020).

\begin{tabular}{|l|l|l|l|l|l|}
\hline \multirow{2}{*}{ Ort } & n Tiere & \multicolumn{3}{|c|}{ n erlegte / gefangene Tiere } & n JAB mit \\
Genehmigung
\end{tabular}

Beseitigung einer invasiven Art besser auf mehrere Strategien zurückgegriffen und nicht auf die Funktionsfähigkeit nur einer einzigen Strategie gesetzt werden sollte. So war in Kosel eine größere Anzahl von Tieren auf eng umgrenzten Raum über einen Zeitraum von mehreren Monaten auch tagsüber und auf kurze Distanz anzutreffen, womit die besten Voraussetzungen für eine Entnahme erfüllt waren. Dennoch sind bei Kosel Muntjaks bisher ausschließlich durch private Nicht-Jäger gefangen worden oder haben sich in Zäunen verfangen und keines wurde mit jagdlichen Methoden erlegt, obwohl die Jagdausübungsberechtigten von Koseler Jagdbezirken über Monate hinweg gültige Entnahmegenehmigungen erhalten hatten. Unklar bleibt dabei, über wie viele Jagdbezirke das Streifgebiet der Muntjaks umfasst hat und wieviele JAB die Gelegenheit zum Abschuss gehabt hätten.

Für zukünftige Fälle sollte also davon ausgegangen werden, dass der Wille, die Fähigkeit oder sonstige Voraussetzungen zur erfolgreichen Beteiligung an Entnahmen nicht bei jedem „Entnahmegenehmigten“ vorhanden ist. Vielmehr sollten parallel weitere Entnahmestrategien angewendet werden oder auch Entnahmen ganz ohne Beteiligung von JAB umgesetzt werden, indem umgehend besonders geeignete Wildtiermanagementexperten beauftragt werden. Eventuell erforderliche Abstimmungen mit den Revierinhabern sollten einfach und schnell möglich sein, um Konflikte mit der Jagdausübung zu vermeiden. Zum einen dürften gerade Jäger großes Verständnis für die Notwendigkeit einer schnellen Entnahme von invasiven Arten in der frühen Phase der Invasion haben und hilfreich zur Seite stehen, zum anderen hat sich im Zuge von z.B. der Entnahmeaktion vom Wolf GW924m in Schleswig-Holstein gezeigt, dass die in einer Region oft namentlich bekannten Jäger bei ethisch besonders kontrovers diskutierten „Beseitigungsaktionen“ zu ihrem eigenen Schutz gar nicht beteiligt sein wollen (Rauterberg et al. 2019).
Auffallend hohe Erfolgsquoten hatte im Koseler Fall hingegen der Einsatz engagierter Nicht-Jäger, die über einige Monate hinweg vor allem durch strategisch günstig aufgestellte Schafzäune gleich mehrere Tiere meist unverletzt einfangen und dann in Abstimmung mit dem LLUR in eine sichere Gehegehaltung überführen konnten. Diese Schafzaun-Methode scheint insbesondere in Siedlungsnähe sicherer und auch im Hinblick auf den Tierschutz vertretbarer zu sein als der Abschuss.

Im Kummerfelder Fall hingegen sind im Vergleich nur sehr wenige (einzelne) Jäger ermächtigt worden, die dortigen Muntjaks zu entnehmen. Aufgrund der nur wenigen Sichtmeldungen aus der Bevölkerung kann angenommen werden, dass dort nur wenige Muntjaks vorkommen. Dennoch haben die wenigen JAB es innerhalb von kurzer Zeit geschafft, vier Muntjaks zu erlegen - hier hat die Beteiligung von JAB gute Erfolge gezeigt. Allerdings könnten auch hier ergänzende Strategien, z.B. durch Netzfang in befriedeten Bereichen, in denen keine Abschüsse möglich sind, dabei helfen, noch mehr Muntjaks der Natur zu entnehmen, denn lt. lokalen Aussagen werden im Siedlungsbereich immer noch Tiere gesichtet.

\section{Muntjaks in anderen Bundesländern}

In Rheinland-Pfalz sind 2018 diverse Muntjaks gesichtet worden und eine Allgemeinverfügung zum Abschuss der Individuen wurde erlassen (Mueef 2018), in 2021 ist dann erneut ein Tier gesichtet und erfolgreich gefangen worden (RP-Online 2021). In 2021 ist in Bayern ein freilebendes Muntjak Opfer eines Verkehrsunfalls geworden, nachdem in den Tagen zuvor ein Muntjak mit Hilfe einer Fotofalle dokumentiert wurde (Führen 2021). Auf einem entsprechenden Portal der Europäischen Union sind weitere Einträge über Meldungen z.B. aus Hessen zu finden (European Commission 2021). 


\section{Fazit für die Praxis}

Muntjaks können große Probleme verursachen, weshalb deren schnelle und vollständige Beseitigung aus der freien Landschaft nicht nur aus ökologisch-ökonomischen Gründen geboten, sondern für Behörden und Jagdrevierinhaber auch rechtlich verpflichtend ist. Die bisherigen Aussetzungen in Schleswig-Holstein dürften bei entsprechend energischem Vorgehen noch $\mathrm{zu}$ beseitigen sein, wodurch die nicht unwahrscheinliche Etablierung und Invasion noch verhindert werden kann. Dafür dürfte die Ermächtigung der Jäger nicht ausreichend sein, sondern weitere effektive Bekämpfungs- und zukünftige Kontrollmethoden müssen herangezogen werden. Weitere Ansiedlungsversuche müssen unbedingt verhindert werden, wobei auch Aufklärungskampagnen helfen dürften.

\section{Danksagung}

Den beiden Gutachtern danken wir für wertvolle Hinweise und Verbesserungen des Manuskripts. Den beteiligten Personen rund um Kosel und Kummerfeld danken wir für zahlreiche Meldungen von Beobachtungen, Fängen und sonstigen Ereignissen und v.a. für die Aktionen vor Ort. Henrik Schulz danken wir für die Fotos, dem LLUR für viele Infos und seine Aktivitäten, dem MELUND für diverse Auskünfte, v.a. über die bestehende und zukünftig mögliche rechtliche Situation. Wir entschuldigen uns bei den Muntjaks, die nichts dafür können, hier von Unbekannten angesiedelt zu werden, aber aus gutem Grund als invasiv gelten. Wir lieben Muntjaks - aber nur im von ihnen selbst bestimmten Areal.

\section{Autoren}

\section{Björn Schulz}

Björn Schulz ist als in Kiel studierter und promovierter Agrarökologe haupt- und ehrenamtlich im Naturschutz in Schleswig-Holstein tätig. Die praktische Wiedervernetzung von Lebensräumen zieht sich als roter Faden durch seine Tätigkeiten. Dabei legt er nach Möglichkeit einen besonderen Schwerpunkt auf ausgewählte (Säuge)Tierarten.

\section{Peter Borkenhagen}

Peter Borkenhagen ist als promovierter Zoologe seit 40 Jahren ehrenamtlich als langjähriges Mitglied der Faunistisch-Ökologischen Arbeitsgemeinschaft und in enger Zusammenarbeit mit dem LLUR mit der faunistischen Erfassung von Säugetieren in Schleswig-Holstein beschäftigt. Seine Schwerpunkte sind gefährdete Arten und die Neozoen.

\section{Literatur}

Annoncen.org (2020) Muntjak, Zwerghirsch, 1.0 günstig abzugeben, http:// schleswig-holstein-sierksdorf.annoncen.org/annonce-750357.html, letzter Zugriff am 19.10.2021.

Baiwy E, Schockert V, Branquart E (2013) Risk analysis of the Reeves' muntjac (Muntiacus reevesi). Risk analysis report of non-native organisms in Belgium. Cellule interdépartementale sur les Espèces invasives (CiEi) DG03. SPW / Editions.

Blottiere D (2018) Sur la présence en France du cerf Muntjac. http:/especes-exotiques-envahissantes.fr/sur-la-presence-en-france-du-cerfmuntjac/, letzter Zugriff 19.10.2021.

Borkenhagen P (2011) Die Säugetiere Schleswig-Holsteins. Husum Druckund Verlagsgesellschaft, Husum.

Boyens Medien (2016) Exotischer Hirsch in Meldorfs Gärten, https://www. boyens-medien.de/artikel/dithmarschen/exotischer-hirsch-in-meldorfer-gaerten.html, letzter Zugriff am 19.10.2021.

Boyens Medien (2017) Muntjak eine invasive Art, https://www.boyens-medien.de/artikel/dithmarschen/meldorf-muntjak-eine-invasive-art.html, letzter Zugriff am 19.10.2021.

Burgin CJ, Wilson DE, Mittermeier RA, Rylands AB, Lacher TE, Sechrest W (2020) Illustrated Checklist of the Mammals of the World. Volume 2: Eulipotyphla to Carnicora. Lynx Edicions, Barcelona.

CABI / Center for Agriculture and Bioscience International (2021): Datasheet Muntjacus reevesi. https://www.cabi.org/isc/datasheet/74281, letzter Zugriff am 19.10.2021.

Casaer J, Boone N, Devisscher S, Vercammen J, Adriaens T (2015) Best practice voor beheer van Chinese muntjak Muntiacus reevesi in Vlaanderen. Rapporten van het Instituut voor Natuur- en Bosonderzoek 2015 (INB0.R.2015.7092003). Instituut voor Natuur- en Bosonderzoek, Brüssel.

Chapman DI, Chapman NG, Dansie 0 (1984) The periods of conception and parturition in feral Reeves' muntjac (Muntiacus reevesi) in southern England, based upon age of juvenile animals. J. Zool. 204: 575-578.

Chapman NG (1996) Reeves' Muntjak (Muntiacus reevesi) in Großbritannien. Zeitschrift für Jagdwissenschaft 42:173-179, doi: 10.1007/ BF02242541.

Chapman NG, Claydon K, Claydon M, Harris S (1987) Techniques for the safe and humane capture of freeliving muntjac deer (Muntiacus reevesi). British Veterinary Journal 143:35-43

Chapman NG, Claydon K, Claydon M, Forde PG, Harris S (1993) Sympatric populations of muntjac (Muntiacus reevesi) and roe deer (Capreolus capreolus): a comparative analysis of their ranging behaviour, social organization and activity. Journal of Zoology 229(4): 623-640, doi: 10.1111/j.1469-7998.1993.tb02660.x.

Clavero M (2014) Shifting Baselines and the Conservation of Non-Native Species. Conservation Biology 28(5): 1434-1436, doi: 10.1111/ cobi. 12266.

Concentus-redivivus.de (2020) Kuriose Hirsche aus China, http://tierparktest.concentus-redivivus.de/wordpress/blog/2015/02/09/muntjaks/, letzter Zugriff am 19.10.2021. 
Cooke AS, Farrell L (2001) Impact of muntjac deer (Muntiacus reevesi) at Monks Wood National Nature Reserve, Cambridgeshire, Eastern England. Forestry 74(3):241-250, doi: 10.1093/forestry/74.3.241.

Cooke AS, Green P, Chapman NG (1996) Mortality in a feral population of muntjac Muntiacus reevesi in England. Acta Theriologica 41 (3):277286.

Diana-Jagdreisen.de (2020) Muntjak, chinesisches Wasserreh und Damhirsch in Bedforshire, https://www.diana-jagdreisen.de/euro$\mathrm{pa} /$ england/muntjak-und-damhirsch-in-england, letzter Zugriff am 19.10.2021.

Dick JTA, Provan J, Reid N (2009) Muntjac Knowledge Transfer: Ecology of introduced muntjac deer and appraisal of control procedures. Report prepared by the Natural Heritage Research Partnership. Quercus for the Northern Ireland Environment Agency, Northern Ireland (UK), doi: 10.13140/RG.2.2.19136.94724

Dolman PM, Wäber K (2008) Ecosystem and competition impacts of introduced deer. Wildlife Research 35:202-214, doi: 10.1071/WR07114

European Commission (2021) European Alien Species Notification System, https://easin.jrc.ec.europa.eu/notsys, letzter Zugriff am 19.10.2021.

Freeman MS, Beatty GE, Dick JTA, Reid N, Provan J (2015) The paradox of invasion: Reeves' muntjac deer invade the British Isles from a limited number of founding females. Journal of Zoology 298(1):54-63, doi: $10.1111 /$ jzo. 12283

Führen K (2021) Muntjak: Überraschende Entdeckung bei Wildunfall, https://www.jagderleben.de/news/muntjak-ueberraschende-entdeckung-wildunfall-712732, letzter Zugriff am 19.10.2021.

Greiser G, Krüger S, Martin I, Thelke F (2020) Status und Entwicklung ausgewählter Wildtierarten in Deutschland. Jahresbericht 2018. Wildtier-Informationssystem der Länder Deutschlands ( WILD). Deutscher Jagdverband, Berlin

Groves C, Grubb C (2011) Ungulate Taxonomy. Johns Hopkins University Press, Baltimore.

Hemami MR, Watkinson AR, Gill RMA, Dolman PM (2007) Estimating abundance of introduced Chinese muntjac Muntiacus reevesi and native roe deer Capreolus capreolus using portable thermal imaging equipment. Mammal Review 37:246-254, doi: 10.1111/j.13652907.2007.00110.x.

Husumer Nachrichten (2020) Jäger warnen: Muntjak bedroht heimische Tierwelt, https://www.shz.de/29184897, letzter Zugriff am 19.10.2021.

Kolligs D (2003) Schmetterlinge Schleswig-Holsteins. Wachholtz, Neumünster.

Langbein J (2007) National Deer-Vehicle Collisions Project - England (2003-2005). Deer Initiative Research Report 7/1, Wrexham.

Meinig H, Boye P, Dähne M, Hutterer R, Lang J (2020) Rote Liste und Gesamtartenliste der Säugetiere (Mammalia) Deutschlands. Bundesamt für Naturschutz, Bonn.

Ministerium für Umwelt, Energie, Ernährung und Forsten (2018) Allgemeinverfügung für den Abschuss von Muntjaks, einer invasiven Art von unionsweiter Bedeutung. Staatsanzeiger für Rheinland-Pfalz 29:769-770

Molinari-Jobin A, Molinari P, Breitenmoser-Würsten C, Wölfl M, Stanisa C Fasel M, Stahl P, Vandel J-M, Rotelli L, Kaczensky P, Huber T, Adamic M, Koren I, Breitenmoser U (2003) The Pan-Alpine Conservation Strategy for the Lynx. Council of Europe Publishing, Straßburg.

Pollard E, Cooke AS (1993) Impact of muntjac deer Muntiacus reevesi on EGG-laying sites of the white admiral butterfly Ladoga camilla in a cambridgeshire wood. Biological Conservation 70 (2):189-191, doi: 10.1016/0006-3207(94)90287-9.

Ogilby (1839) Muntiacus reevesi. In: GBIF | Global Biodiversity Information Facility, https://www.gbif.org/species/2440946, letzter Zugriff am 19.10.2021.
Quoka.de (2020) Suche Muntjaks zu kaufen zur tiergerechten Haltung in weitläufigem Gehege, https://m.quoka.de/tiermarkt/muntjak/kappeln/sc_48_ct_116347.html, letzter Zugriff am 23.6.2020.

Rauterberg C, Hotsch N, Jacobsen J (2019) Problemwolf - Jagd macht Jägern Probleme, https://www.ndr.de/nachrichten/schleswig-holstein/Problemwolf-Jagd-macht-Jaegern-Probleme, problemwolf118. html, letzter Zugriff am 16.10.2020.

RP-Online (2021) Seltener Muntjak-Hirsch ist eingefangen, https:// $\mathrm{rp}$-online.de/nrw/staedte/viersen/kreis-viersen-seltener-hirsch-isteingefangen_aid-58312295, letzter Zugriff am 19.10.2021.

Tierparkgettorf.de (2020) Indischer Muntjak Muntjacus muntjak, https:// tierparkgettorf.de/tiere-und-park/tierlexikon/details/indischermuntjak.html, letzter Zugriff am 19.10.2021

Timmins J, Chan B (2016) Muntiacus reevesi. The IUCN Red List of Threatened Species 2016, https://www.iucnredlist.org/species/42191/170905827, letzter Zugriff am 19.10.2021.

Wagener MG, Henniger T, Molnár V, Ganter M (2019) Der Muntjak - Steckbrief einer invasiven Art. Deutsches Tierärzteblatt 67 (9):1240-1246.

Wildtierkataster SH (2020) Die Fauna (Tierwelt) Schleswig-Holsteins, https://www.wildtier-kataster.uni-kiel.de/pages/tierarten.php, letzter Zugriff am 19.10.2021

WildundHund.de (2018) Muntjac in Rheinland-Pfalz - Forumsbeiträge div. Mitglieder, https://forum.wildundhund.de/threads/muntjac-inrheinland-pfalz.118215/, letzter Zugriff am 19.10.2021.

Ziehm R (2015) Muntjak-Hirsch stromert durch Tungendorfs Gärten, https://www.shz.de/10806656, letzter Zugriff am 19.10.2021.

\section{Open Access}

>> Der Artikel ist unter der Creative-Commons-Lizenz Namensnennung 4.0 International veröffentlicht. Den Vertragstext finden Sie unter: https://creativecommons.org/licenses/by/4.0/deed.de. Bitte beachten Sie, dass einzelne, entsprechend gekennzeichnete Teile des Artikels von der genannten Lizenz ausgenommen sein bzw. anderen urheberrechtlichen Bedingungen unterliegen können. 